\title{
RETIFICADOR TRIFÁSICO ISOLADO COM CORREÇÃO DO FATOR DE POTÊNCIA EMPREGANDO O CONVERSOR CC-CC SEPIC EM CONDUÇÃO CONTÍNUA
}

\author{
Denizar Cruz Martins, Anderson H. de Oliveira e Ivo Barbi \\ Universidade Federal de Santa Catarina - INEP - Instituto de Eletrônica de Potência \\ Fone:(048) 331.9204 Fax:(048) 234.5422 e-mail: denizar@inep.ufsc.br \\ Cx.Postal 5119 CEP:88.040-970 Florianópolis - SC
}

\begin{abstract}
Resumo - Neste artigo é proposto um retificador trifásico utilizando o conversor CC-CC SEPIC operando no modo de condução contínua para a correção do fator de potência. As principais características desta estrutura são: simplicidade e robustez do circuito de potência, possibilidade de operação como elevadora ou abaixadora de tensão, baixa distorção harmônica da corrente de entrada, ser naturalmente isolada e processar toda a energia em um único estágio, utilizando apenas uma chave controlada. $O$ conversor opera à freqüência constante e a transferência de energia para a carga é controlada através da modulação PWM. É feito um estudo da estrutura em regime permanente e apresentado um procedimento de projeto. Os resultados teóricos são comprovados através de um protótipo de laboratório.
\end{abstract}

Abstract - This paper presents an analysis of a threephase rectifier with high power factor using a SEPIC DCDC converter operating in continuous conduction mode (CCM). The structure is particularly simple and robust. Its main features are: one power processing stage, which can operate as step-down or step-up converter, lower harmonic distortion in the line current and natural isolation. The converter works with constant frequency and PWM modulation. A study for steady state conditions, a design procedure, and experimental results obtained from a laboratory prototype are presented.

\section{INTRODUÇÃO}

Os sistemas de alimentação trifásico, disponíveis em aplicações industriais, geralmente são mais indicados para sistemas de potências elevadas (acima de $1 \mathrm{~kW}$ ), onde a conversão $\mathrm{CA} / \mathrm{CC}$ tem sido dominada por retificadores convencionais a diodos e tiristores. A característica não ideal da corrente de entrada destes retificadores cria problemas para a rede comercial de energia elétrica, dentre os quais podem ser destacadas:

- Distorção da tensão de alimentação devido aos altos picos de corrente requeridos pelo retificador, podendo prejudicar o funcionamento de outros equipamentos conectados ao mesmo ponto;

- Interferência eletromagnética nos sistemas de comunicação e controle;

- Aumento das perdas nos elementos das redes de transmissão e distribuição;

- Redução do fator de potência na entrada do estágio retificador;
- Necessidade de geração de grandes quantidades de potência reativa, elevando os custos de todo o sistema;

- Diminuição do rendimento da estrutura devido ao elevado valor eficaz da corrente de entrada do retificador.

Muitos trabalhos têm sido apresentados pela comunidade científica a fim de proporcionar a utilização de retificadores trifásicos com fator de potência unitário e baixo conteúdo harmônico na corrente de linha [1-7]. Uma das estruturas mais empregadas, como pré-regulador, é o conversor Boost [1,2]. Essa estrutura não é naturalmente isolada, somente opera como elevador de tensão e trabalha no modo de condução descontinua. O conversor proposto em [2] tem um bom desempenho, mas sua estrutura é composta de três interruptores sincronizados, três indutores BuckBoost conectados em Y, e um interruptor adicional para controle da tensão contínua de saída. Além disso o conversor opera em condução descontínua com elevado valor eficaz de corrente. Em [4] as principais vantagens são a simplicidade e o bom desempenho da estrutura; contudo, o sistema opera em condução descontínua e apresenta elevados esforços de corrente nas chaves. Os trabalhos apresentados em $[5,6]$ são muito interessantes, e apresentam um fluxo constante de energia. Entretanto, os circuitos de comando e controle são excessivamente complexos, e particularmente em [5] a transferência de energia é processada em duas etapas. Os conversores não são independentes e, por essa razão, a confiabilidade do sistema fica comprometida. Na referência [7] são obtidos excelentes resultados em termos do rendimento do sistema, operando com fluxo constante de energia, mas há grandes dificuldades em nível de implementação dos circuitos de comando, devido sua complexidade e transferência de energia em dois estágios. Diante desses inconvenientes e procurando melhorar a performance dos conversores CA-CC de alta potência, este artigo apresenta a análise e o desenvolvimento de um retificador trifásico, com alto fator de potência, operando com frequiência constante, com um único estágio de processamento de energia, e empregando o conversor CC-CC Sepic em condução contínua. A estrutura proposta é naturalmente isolada, e utiliza apenas uma chave para o controle do fluxo de potência, tornando o circuito de comando extremamente simples, e não necessita de filtros de linha entre a rede e o retificador. Além disso, o sistema pode operar como elevador ou abaixador de tensão, e o reduzido número de componentes aumenta a confiabilidade do sistema, tornando-o bastante atraente para aplicações industriais de alta potência. 


\section{CIRCUITO PROPOSTO E PRINCÍPIO DE OPERAÇÃO}

O circuito de potência do retificador proposto é apresentado na Figura 1. A fim de facilitar o estudo da estrutura, serão consideradas as seguintes simplificações:

- A análise é feita para o circuito operando em regime permanente;

- Todos os componentes são considerados ideais;

- O transformador é representado pela sua indutância magnetizante referida ao lado primário;

- As ondulações de tensão nos capacitores C1 e Co são consideradas nulas;

- As tensões de rede são consideradas constantes em um período de chaveamento do conversor.

Referindo os parâmetros secundários do conversor para o lado primário tem-se o circuito equivalente da Figura 2, onde Vin $=2,34 . V_{\text {leficaz }}$ e:

$\mathrm{Ro}=\left(\frac{\mathrm{Np}}{\mathrm{Ns}}\right)^{2} \cdot \mathrm{Ro}^{\prime} ; \mathrm{Co}=\left(\frac{\mathrm{Ns}}{\mathrm{Np}}\right)^{2} \cdot \mathrm{Co}^{\prime} ; \mathrm{Vo}=\frac{\mathrm{Np}}{\mathrm{Ns}} \cdot \mathrm{Vo}^{\prime}$

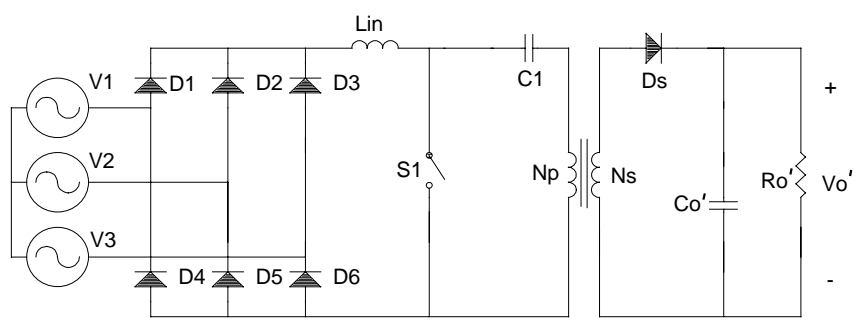

Figura 1: Diagrama básico do retificador trifásico utilizando o conversor CC/CC Sepic.

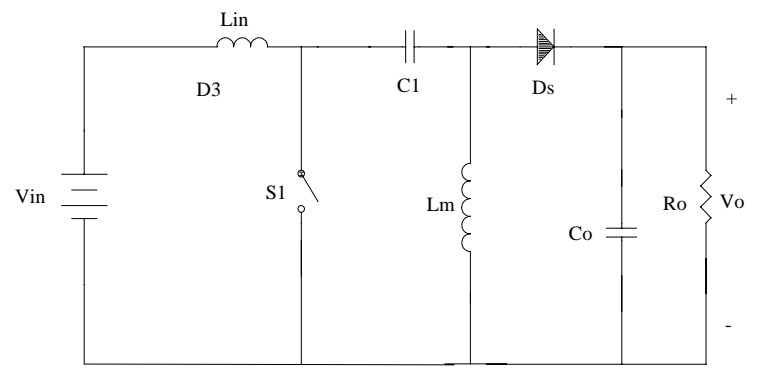

Figura 2: Diagrama básico do retificador trifásico utilizando o conversor CC/CC Sepic, com os parâmetros referenciados ao lado primário do transformador.

O conversor Sepic operando em condução contínua apresenta duas etapas de operação, mostradas a seguir:

- 1- etapa : intervalo $(0<\mathrm{t}<\mathrm{D}$.T $)$. Nesta etapa a chave $\mathrm{S} 1$ está conduzindo. A energia proveniente da rede de alimentação é armazenada no indutor de entrada Lin e o capacitor C1 transfere energia para a indutância magnetizante Lm. A tensão no capacitor $\mathrm{C} 1$ é considerada constante e igual a Vin, ela representa a tensão média de um retificador trifásico convencional. As correntes $i_{e}$ e $i_{L m}$ crescem linearmente com uma taxa de variação igual a Vin/Lin e Vin/Lm respectivamente. Durante esta etapa, o diodo Ds permanece bloqueado e o capacitor Co fornece energia para a carga Ro. O circuito equivalente desta etapa é mostrado na Figura 3.

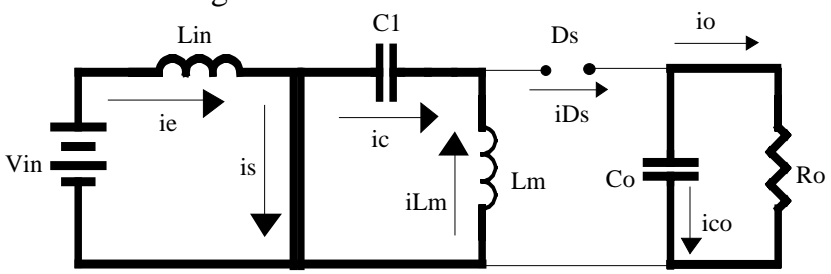

Figura 3: Primeira etapa.

- $2^{\mathbf{a}}$ etapa : intervalo (D. $\mathrm{T}<\mathrm{t}<\mathrm{T}$ ). Nesta etapa a chave $\mathrm{S} 1$ é bloqueada e o diodo Ds passa a conduzir, transferindo a energia armazenada nos indutores para a carga Ro. As correntes $i_{e}$ e $i_{L m}$ decrescem linearmente com uma taxa de variação igual a $\mathrm{Vo} / \mathrm{Lin}$ e $\mathrm{Vo} / \mathrm{Lm}$ respectivamente. Durante esta etapa o capacitor $\mathrm{C} 1$ acumula energia. O circuito equivalente é mostrado na Figura 4.

O modo de condução contínua é caracterizado pela habilitação da chave S1 a conduzir antes que a corrente no diodo Ds se anule. As formas de onda deste modo de operação são mostradas na Figura 5.

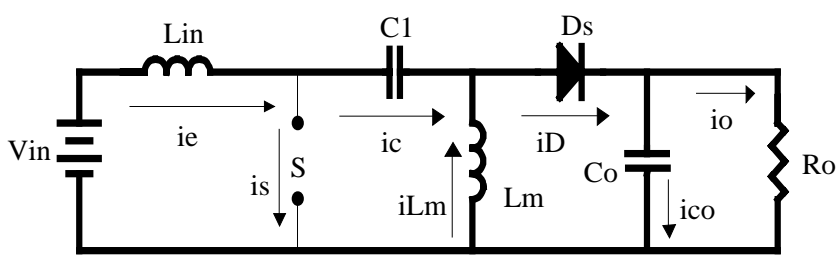

Figura 4: Segunda etapa.

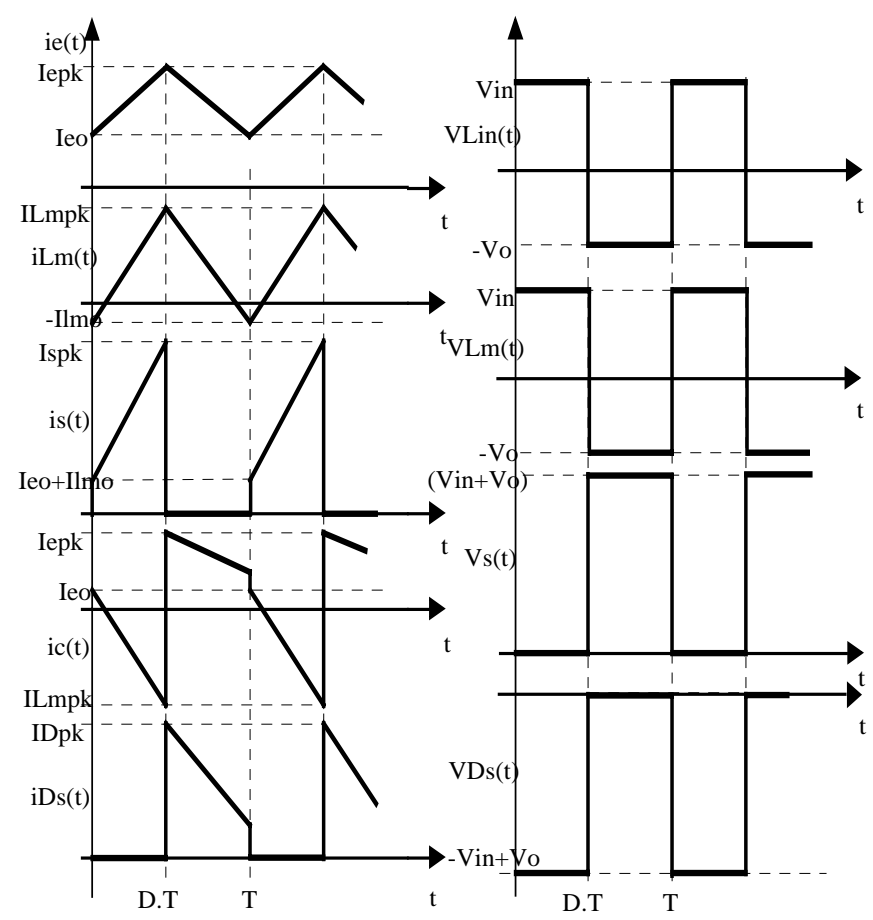

Figura 5: Principais formas de onda. 


\section{ANÁLISE QUANTITATIVA}

As equações que regem o funcionamento do conversor Sepic em condução contínua e em regime permanente são dadas a seguir.

$$
\begin{aligned}
& \mathrm{ie}(\mathrm{t})=\mathrm{Ie}_{\mathrm{o}}+\frac{\mathrm{Vin}}{\mathrm{Lin}} \cdot \mathrm{t} \text { para } 0<\mathrm{t}<\mathrm{D} . \mathrm{T} \\
& \mathrm{ie}(\mathrm{t})=\frac{\mathrm{Vin}}{\mathrm{Lin}} \cdot \mathrm{D} \cdot \mathrm{T}-\frac{\mathrm{Vo}}{\mathrm{Lin}} \cdot(\mathrm{t}-\mathrm{D} \cdot \mathrm{T})+\mathrm{Ie}_{\mathrm{o}} \\
& \text { para } \mathrm{D} . \mathrm{T}<\mathrm{t}<\mathrm{T} \\
& \operatorname{iLm}(\mathrm{t})=\mathrm{I}_{\mathrm{Lm}_{\mathrm{O}}}+\frac{\mathrm{Vin}}{\mathrm{Lm}} \cdot \mathrm{t} \quad \text { para } 0<\mathrm{t}<\mathrm{D} . \mathrm{T} \\
& \operatorname{iLm}(t)=\frac{V i n}{L m} \cdot D \cdot T-\frac{V o}{L m}(t-D . T)+I_{L_{O}} \\
& \text { para D. T }<\mathrm{t}<\mathrm{T}
\end{aligned}
$$

is $(t)=\frac{\text { Vin }}{\text { Leq }} \cdot t+\mathrm{Ie}_{\mathrm{O}}+\mathrm{I}_{\mathrm{Lm}_{\mathrm{O}}} \quad$ para $0<\mathrm{t}<\mathrm{D} . \mathrm{T}$

is $(\mathrm{t})=0 \quad$ para D.T $<\mathrm{t}<\mathrm{T}$

$\mathrm{ic}_{1}(\mathrm{t})=-\frac{\mathrm{Vin}}{\mathrm{Lm}} \cdot \mathrm{t}-\mathrm{I}_{\mathrm{Lm}_{\mathrm{O}}} \quad$ para $0<\mathrm{t}<\mathrm{D} . \mathrm{T}$

$\mathrm{ic}_{1}(\mathrm{t})=\frac{\mathrm{Vin}}{\mathrm{Lin}} \cdot \mathrm{D} \cdot \mathrm{T}-\frac{\mathrm{Vo}}{\operatorname{Lin}}(\mathrm{t}-\mathrm{D} \cdot \mathrm{T})+\mathrm{Ie}_{\mathrm{o}} \quad$ para D.T $<\mathrm{t}<\mathrm{T}$

$$
\begin{aligned}
& \operatorname{iDs}(t)=0 \text { para } 0<\mathrm{t}<\text { D.T } \\
& \operatorname{iDs}(\mathrm{t})=\frac{\mathrm{Vin}}{\mathrm{Leq}} \cdot \mathrm{D} \cdot \mathrm{T}-\frac{\mathrm{Vo}}{\mathrm{Leq}}(\mathrm{t}-\mathrm{D} \cdot \mathrm{T})+\mathrm{Ie}_{\mathrm{O}}+\mathrm{I}_{\mathrm{Lm}_{\mathrm{O}}}
\end{aligned}
$$$$
\text { para D.T }<\mathrm{t}<\mathrm{T}
$$

$\operatorname{VLin}(\mathrm{t}), \operatorname{VLm}(\mathrm{t})=\operatorname{Vin} \quad$ para $0<\mathrm{t}<\mathrm{D}$. T

$\operatorname{VLin}(\mathrm{t}), \operatorname{VLm}(\mathrm{t})=-\mathrm{Vo} \quad$ para D. T $<\mathrm{t}<\mathrm{T}$

$\mathrm{Vs}(\mathrm{t})=0 \quad$ para $0<\mathrm{t}<\mathrm{D} . \mathrm{T}$

$\mathrm{Vs}(\mathrm{t})=\mathrm{Vin}+\mathrm{Vo} \quad$ para D. $\mathrm{T}<\mathrm{t}<\mathrm{T}$

$\operatorname{vDs}(t)=-(\operatorname{Vin}+\operatorname{Vo}) \quad$ para $0<\mathrm{t}<\mathrm{D} . \mathrm{T}$

$\operatorname{VDs}(\mathrm{t})=0 \quad$ para $\mathrm{D} . \mathrm{T}<\mathrm{t}<\mathrm{T}$

onde: $\quad$ Leq $=\frac{\text { Lin. } \mathrm{Lm}}{\operatorname{Lin}+\mathrm{Lm}} ; \mathrm{Vin}=2,34 \cdot \mathrm{V}_{1}$ eficaz ;

D.T é o intervalo de condução da chave S1.

Definindo as ondulações de corrente na entrada $\left(\Delta \mathrm{i}_{\mathrm{e}}\right)$ e na magnetizante do transformador $\left(\Delta \mathrm{i}_{\mathrm{Lm}}\right)$, conforme Figura 6 , é possível se calcular as correntes médias e eficazes nos componentes do conversor Sepic [8].

Obtém-se então:

- Correntes médias de entrada, $\mathrm{Ie}_{\mathrm{md}}$, e na chave $\mathrm{S} 1$, Is $\mathrm{md}_{\mathrm{md}}$ :

$$
\text { Iemd }=\text { Ismd }=\frac{\text { Vin. D. T }}{2 . \overline{\Delta i e} \cdot \text { Lin }}
$$

onde: $\quad \overline{\Delta \mathrm{ie}}=\frac{\Delta \mathrm{ie} / 2}{\text { Iemd }}$

- Correntes médias na indutância magnetizante, $\mathrm{I}_{\text {Lmmd }}$, e no diodo Ds, $\mathrm{I}_{\text {Dsmd }}$ :
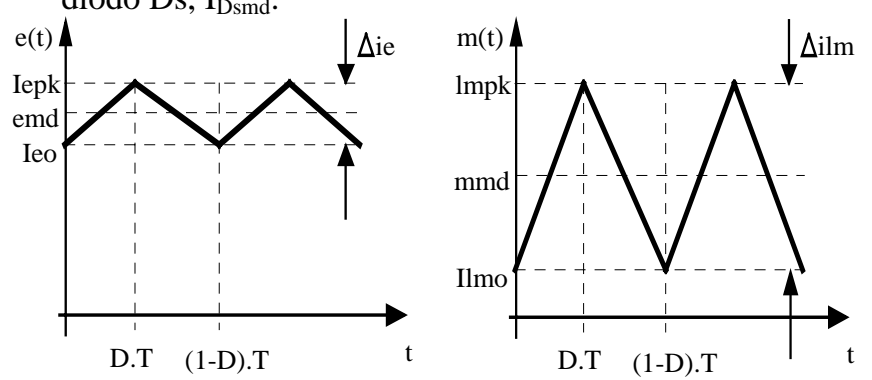

Figura 6: Detalhe das correntes na entrada e na magnetizante do transformador do conversor Sepic.

$$
\mathrm{I}_{\mathrm{Lm}_{\mathrm{md}}}=\mathrm{I}_{\mathrm{Ds} \mathrm{md}}=\frac{\text { Vo.(1-D).T }}{2 \cdot \overline{\Delta \mathrm{iLm} \cdot \mathrm{Lm}}}
$$

onde:

$$
\overline{\Delta \mathrm{iLm}}=\frac{\Delta \mathrm{iLm} / 2}{\mathrm{I}_{\mathrm{Lmmd}}}
$$

- Corrente média nos diodos retificadores, $\mathrm{I}_{\text {Drmd }}$ :

$$
\mathrm{I}_{\text {Drmd }}=\frac{\text { Iemd }}{3}
$$

- Corrente eficaz de entrada $\mathrm{Ie}_{\mathrm{ef}}$ :

$$
\text { Ieef }=\frac{\sqrt{3}}{3} \cdot \frac{\text { Vin. D. T }}{2 \cdot \overline{\Delta \mathrm{ie} \cdot \mathrm{Lin}}} \cdot \sqrt{3+(\overline{\mathrm{\Delta i \textrm {e }}})^{2}}
$$

- Corrente eficaz na chave $\mathrm{S} 1$, $\mathrm{Is}_{\mathrm{ef}}$ :

$$
\text { Isef }=\frac{\sqrt{3}}{3} \cdot \frac{\text { Vin. D. T }}{2 \cdot \overline{\Delta i \mathrm{ie}} \cdot \operatorname{Lin}} \cdot \frac{\sqrt{(\overline{\Delta \mathrm{ie}} \cdot \mathrm{D}+\overline{\Delta \mathrm{iLm}} \cdot(1-\mathrm{D}))^{2}+3}}{\sqrt{\mathrm{D}}}
$$

- Corrente eficaz no capacitor $\mathrm{C} 1, \mathrm{Ic}_{1 \mathrm{ef}}$ :

$$
\mathrm{Ic}_{1} \mathrm{ef}=\frac{\mathrm{Vin} \cdot \sqrt{\mathrm{D}} \cdot \mathrm{T}}{2 \cdot \overline{\Delta \mathrm{ie}} \cdot \operatorname{Lin}} \sqrt{\frac{\left.(1-\mathrm{D}) \cdot\left[\mathrm{D} \cdot(\overline{\Delta \mathrm{ie}})^{2}+(1-\mathrm{D}) \cdot(\overline{\Delta \mathrm{iLm}})^{2}+3\right)\right]}{3}}
$$

- Corrente eficaz no diodo Ds, $\mathrm{I}_{\text {Dsef: }}$ :

$$
\mathrm{I}_{\text {Dsef }}=\frac{\mathrm{Vo} \cdot \sqrt{(1-\mathrm{D})} \cdot \mathrm{T}}{2 \cdot \sqrt{3} \cdot \overline{\Delta \mathrm{iLm} \cdot \mathrm{Lm}}} \cdot\left(\sqrt{(\mathrm{D} \cdot \overline{\Delta \mathrm{ie}}+(1-\mathrm{D}) \cdot \overline{\Delta \mathrm{iLm}})^{2}+3}\right)
$$

- Corrente eficaz no capacitor $\mathrm{Co}, \mathrm{Ic}_{\mathrm{Oef}}$ :

$$
\mathrm{Ic}_{\mathrm{o}} \mathrm{ef}=\frac{\text { Vo. }(1-\mathrm{D}) \cdot \mathrm{T}}{2 \cdot \overline{\Delta \mathrm{iLm}} \cdot \mathrm{Lm}} \cdot \sqrt{\left(\frac{(\mathrm{D} \cdot \overline{\Delta \mathrm{ie}}+(1-\mathrm{D}) \cdot \overline{\Delta \mathrm{iLm}})^{2}+3}{3 \cdot(1-\mathrm{D})}\right)-1}
$$

- Corrente eficaz nos diodos retificadores, $\mathrm{IDr}_{\mathrm{ef}}$ :

$$
\mathrm{I}_{\mathrm{Dr}} \mathrm{ef}=\frac{\sqrt{3+(\overline{\Delta \mathrm{ie}})^{2}}}{3} \cdot \text { Iemd }
$$


- Corrente eficaz em cada fase de entrada, $\mathrm{If}_{\mathrm{ef}}$ :

$$
\text { Ifef }=\sqrt{2} \cdot \frac{\sqrt{3+(\overline{\Delta \mathrm{ie}})^{2}}}{3} \text {. Iemd }
$$

Através da conservação de fluxo magnético do transformador em regime permanente, tem-se que:

$$
\text { Vin. D. T }=\text { Vo. }(1-\mathrm{D}) . \mathrm{T}
$$

Portanto, a característica de transferência estática do conversor Sepic em condução contínua, mostrada na Figura 7, é dada por:

$$
\frac{V_{o}}{V i n}=\frac{D}{(1-D)}
$$

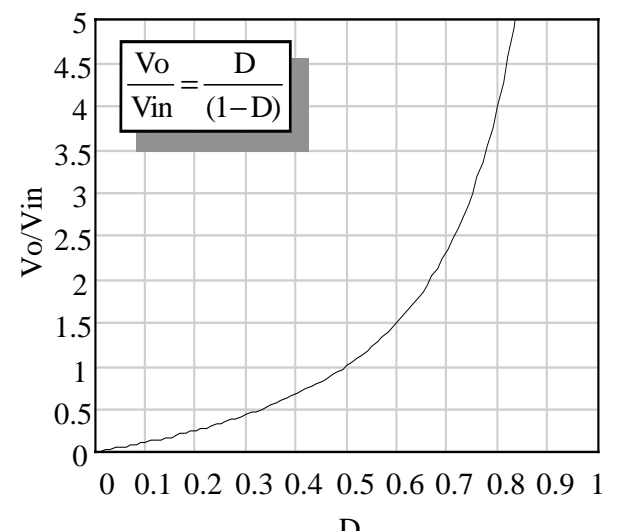

Figura 7: Característica de transferência estática do conversor Sepic em condução contínua.

A Figura 8 apresenta o gráfico das características externas do conversor Sepic em regime permanente [8]. A partir deste gráfico pode-se calcular o valor da corrente de carga crítica que delimita as regiões de condução contínua/descontínua.

\section{PROCEDIMENTO DE PROJETO}

Parametrizando-se as equações apresentadas no item anterior, podem ser gerados ábacos que facilitam o projeto do conversor. Estes ábacos são apresentados juntamente com o procedimento de projeto.

\section{A. Dados iniciais}

Devem ser fornecidos os seguintes dados para que seja feito o projeto do conversor:

- Tensão de fase da rede $\mathrm{V}_{\text {fase }}: 220$ [V]

- Tensão de saída Vo: 120 [V]

- Potência de saída Po: 3.000 [W]

- Frequiência de chaveamento fs: $20[\mathrm{kHz}]$

- Razão cíclica nominal D: 0,4.

- Rendimento $\eta: 90 \%$

\section{B. Ondulação da corrente no indutor de entrada}

A ondulação da corrente no indutor de entrada Lin afeta diretamente $o$ fator de potência (FP) e a taxa de distorção harmônica (THD) da corrente de entrada do conversor.
Portanto, para se obter um fator de potência acima de 0,95 e uma taxa de distorção harmônica próximo a 30\% deve-se escolher a ondulação $\overline{\Delta \mathrm{ie}}$ menor que $10 \%$. Neste projeto

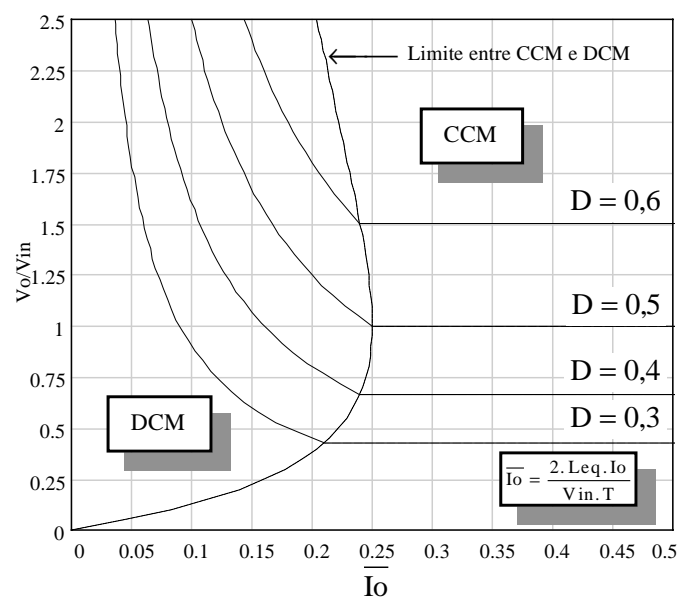

Figura 8: Características externas do conversor Sepic em regime permanente.

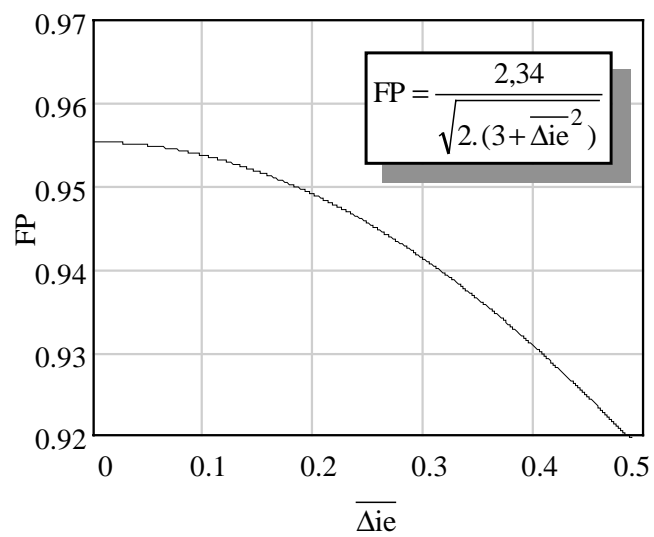

Figura 9: Fator de potência do retificador trifásico em função da ondulação de corrente de entrada.

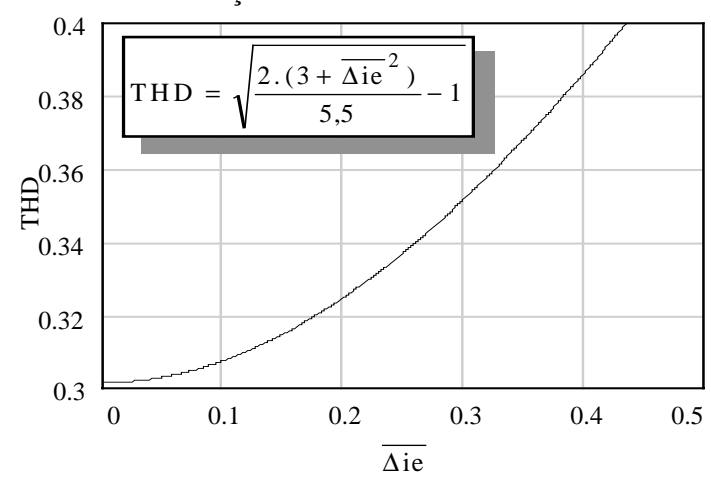

Figura 10: Taxa de distorção harmônica (THD) em função da ondulação de corrente de entrada.

adotou-se $\overline{\Delta \mathrm{ie}}=2,5 \%$ (Veja as Figuras 9 e 10).

C. Relação de transformação do transformador

A relação de transformação do transformador é dada por:

$$
\mathrm{N}=\frac{\text { Vin. } \mathrm{D}}{\operatorname{Vo} \cdot(1-\mathrm{D})}
$$




$$
\text { Logo: } \quad \mathrm{N}=\frac{2,34 \cdot 220 \cdot 0,4}{120 \cdot(1-0,4)}=2,86
$$

D. Cálculo do indutor de entrada Lin

Para se calcular o valor do indutor de entrada deve-se determinar a corrente média de entrada. Desse modo, tem-se:

$$
\text { Iemd }=\frac{\text { Po }}{\eta \cdot \text { Vin }}
$$

Portanto: $\quad$ Iemd $=\frac{3000}{0,9 \cdot 2,34.220}=6,5 \quad[\mathrm{~A}]$

O valor de Lin é obtido através de (10), resultando em:

$$
\text { Lin }=\frac{\text { Vin. D }}{2 \cdot \overline{\Delta \text { ie } . \text { Iemd. fs }}}
$$

Portanto: $\quad$ Lin $=\frac{2,34 \cdot 220 \cdot 0,4}{2 \cdot 0,025 \cdot 6,5 \cdot 20000}=31,68[\mathrm{mH}]$

\section{E. Cálculo da indutância magnetizante do transformador}

Para que seja calculado o valor da indutância magnetizante do transformador (referenciado ao primário), é necessário definir o valor da resistência de carga máxima que garanta o modo de condução contínua do conversor. Através do gráfico da Figura 8 observa-se que o valor de corrente de carga normalizada crítica para $\mathrm{D}=0,4$ é igual a aproximadamente 0,24. Adotando-se o valor de $\overline{\text { Io nominal }}$ igual a 6 vezes maior que $\overline{\text { Io }}$ crítico, obtém-se:

$$
\text { Leq }=\frac{2,34 . \text { Vfase. Vo. N. } \overline{\text { Io }}}{2 . \text { fs. Po }}
$$

Portanto: $\quad$ Leq $=\frac{2,34 \cdot 220 \cdot 120 \cdot 2,86 \cdot 6 \cdot 0,24}{2 \cdot 20000 \cdot 3000}=2,12 \quad[\mathrm{mH}]$

mas:

$$
\text { Leq }=\frac{\text { Lin. } \mathrm{Lm}}{\mathrm{Lin}+\mathrm{Lm}}
$$

Portanto:

$$
\mathrm{Lm}=2,27 \quad[\mathrm{mH}]
$$

\section{F. Cálculo dos capacitores $C 1$ e Co}

Considerando-se a ondulação de tensão nos terminais dos capacitores em torno de $1 \%$ de seu valor médio, obtém-se:

$$
\mathrm{C} 1=\frac{\mathrm{D}^{2} \cdot \mathrm{Po}}{0,01 \cdot(1-\mathrm{D}) \cdot \mathrm{Vo}^{2} \cdot \mathrm{fs} \cdot \mathrm{N}^{2}}
$$

Portanto: $\mathrm{C} 1=\frac{0,4^{2} \cdot 3000}{0,01 \cdot(1-0,4) \cdot 120^{2} \cdot 20000 \cdot 2,86^{2}} \approx 33,96[\mu \mathrm{F}]$

$$
\mathrm{Co}=\frac{\mathrm{D}^{2} \cdot \mathrm{Vin} \cdot \mathrm{Po}}{0,01 \cdot \mathrm{Vo}^{3} \cdot(1-\mathrm{D}) \cdot \mathrm{fs} \cdot \mathrm{N}}
$$

Portanto: $\quad \mathrm{Co}=\frac{0,4^{2} \cdot 2,34 \cdot 220 \cdot 3000}{0,01 \cdot 120^{3} \cdot(1-0,4) \cdot 20000 \cdot 2,86} \cong 417[\mu \mathrm{F}]$

Para facilitar a escolha dos capacitores, são apresentadas nas Figuras.11 e 12, as suas correntes eficazes parametrizadas. A partir dessas figuras, considerando $\mathrm{D}=0,4$, obtém-se:

$$
\begin{aligned}
& \mathrm{Ic}_{1} \mathrm{ef}=1,25 \cdot \mathrm{Iemd}=1,25 \cdot 6,5=8,13[\mathrm{~A}] \\
& \text { Icoef }=0,83 . \text { Iomd }=0,83 \cdot 25=20,75[\mathrm{~A}]
\end{aligned}
$$

\section{G. Escolha dos semicondutores}

Os ábacos das Figuras 13, 14 e 15 auxiliam na escolha da

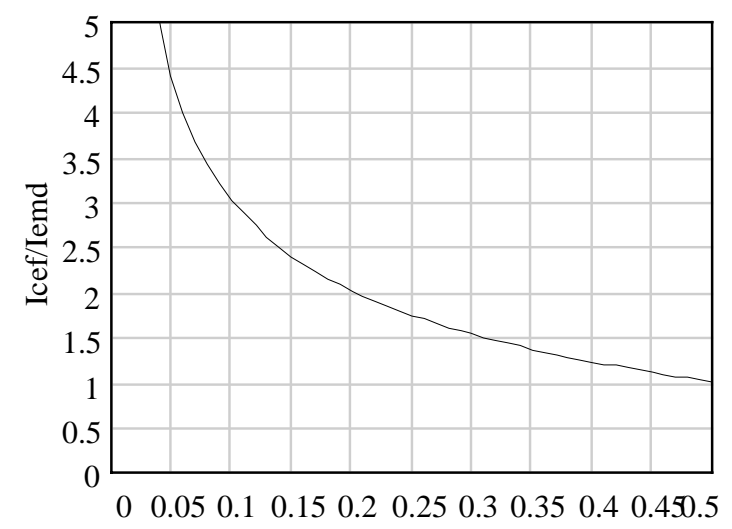

$\mathrm{D}$

Figura 11: Corrente eficaz no capacitor $\mathrm{C} 1$ parametrizada, em função de D.

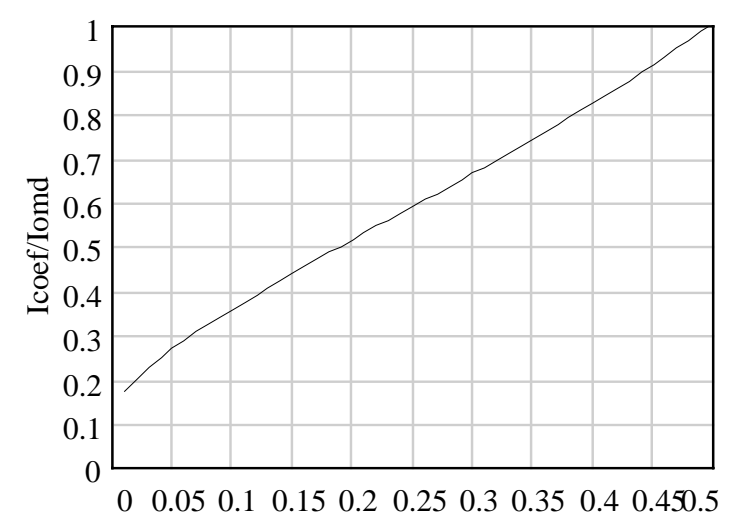

$\mathrm{D}$

Figura 12: Corrente eficaz no capacitor Co parametrizada, em função de D.

chave S1, diodo Ds e diodos retificadores. Eles foram obtidos a partir das equações (10), (11), (12), (14), (16) e (18). Assim, para $\mathrm{D}=0,4$, os valores de pico e eficazes das correntes nos semicondutores podem ser determinados. Portanto:

$$
\begin{aligned}
& \text { Ispk }=3,0 . \text { Iemd }=3,0.6,5=19,5[\mathrm{~A}] \\
& \text { Isef }=1,6 . \text { Iemd }=1,6 \cdot 6,5=10,4[\mathrm{~A}] .
\end{aligned}
$$

$\mathrm{I}_{\mathrm{Ds}} \mathrm{pk}=1,88$. Iomd $=1,88.25=47$ [A]

$$
\begin{aligned}
& \mathrm{I}_{\mathrm{Ds}} \text { ef }=1,3 \cdot \mathrm{Iomd}=1,3 \cdot 25=32,5[\mathrm{~A}] . \\
& \mathrm{I}_{\mathrm{Dr}} \mathrm{pk}=3,1 \cdot \mathrm{I}_{\mathrm{Dr}} \mathrm{md}=3,1 \cdot 2,17=6,73[\mathrm{~A}] \\
& \mathrm{I}_{\text {Dr }} \text { ef }=1,735 \cdot \mathrm{I}_{\mathrm{Dr}} \mathrm{md}=1,735 \cdot 2,17=3,76[\mathrm{~A}] .
\end{aligned}
$$

Com estes valores todos os componentes do circuito de potência podem ser dimensionados.

As Figuras 11, 13, 14 e 15 podem ser consideradas aproximações aceitáveis frente à pequena variação de Vin a cada período da rede. 


\section{RESULTADOS EXPERIMENTAIS}

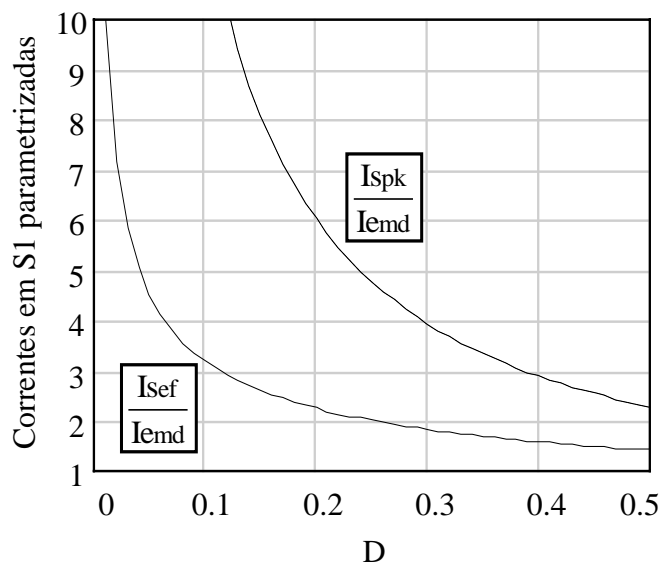

Figura 13: Corrente eficaz e de pico na chave S1, parametrizadas, em função de D.

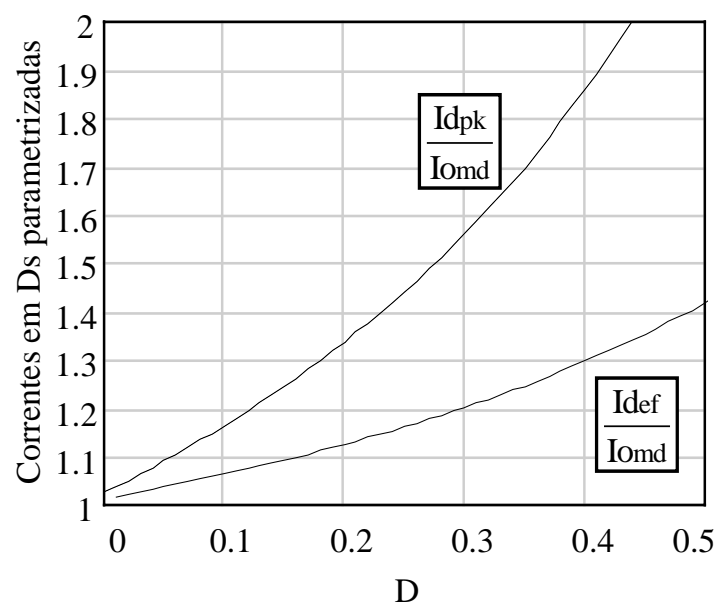

Figura 14: Corrente eficaz e de pico em Ds, parametrizadas, em função de D.
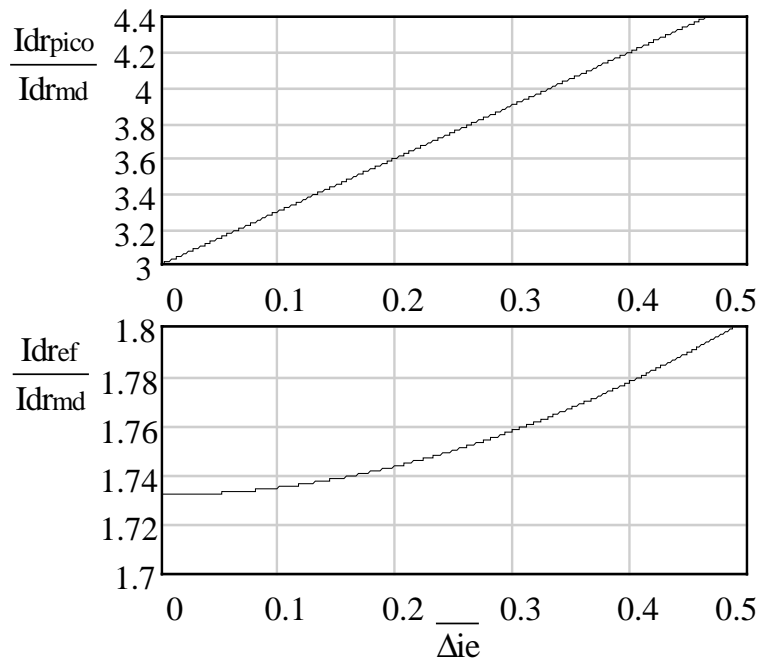

Figura 15: Corrente eficaz e de pico em Dr, parametrizadas, em função de $\overline{\Delta \mathrm{ie}}$.
Para comprovar a validade do procedimento de projeto apresentado no item anterior, foi montado um protótipo de laboratório de 3,0 kW. As principais especificações foram dadas no item IV.A. Todos os resultados apresentados neste trabalho foram obtidos para a condição de plena carga (exceto as Figuras 20,21,22), e a tensão de saída foi mantida constante igual a $120 \mathrm{~V}$. A Figura 16 mostra a tensão e a corrente na fase 1 da rede elétrica. A tensão e a corrente na chave principal S1 está mostrada na Figura 17. A Figura 18 apresenta a tensão e a corrente no diodo Ds. A corrente no indutor de entrada é apresentada na Figura 19.

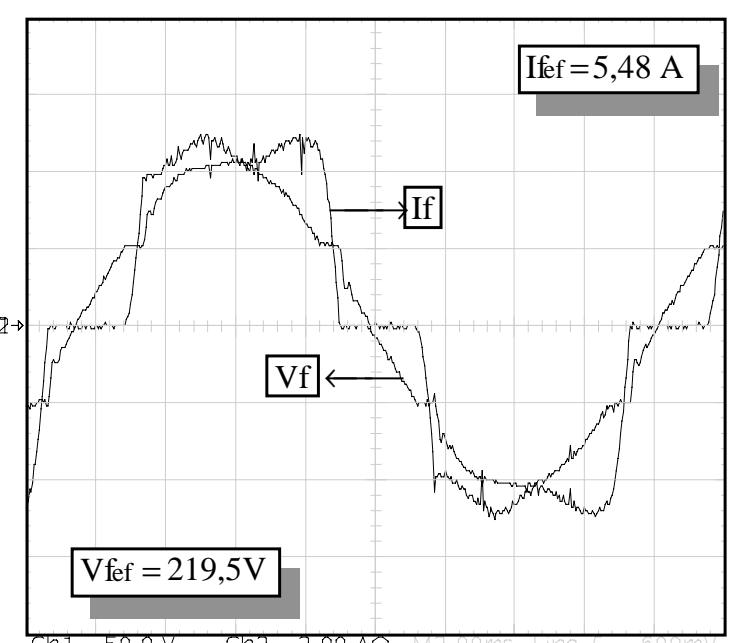

Figura 16: Tensão e corrente de fase na entrada do retificador. Escala: $150 \mathrm{~V} / \mathrm{div} ; 3 \mathrm{~A} / \mathrm{div} ; 2 \mathrm{~ms} / \mathrm{div}$.

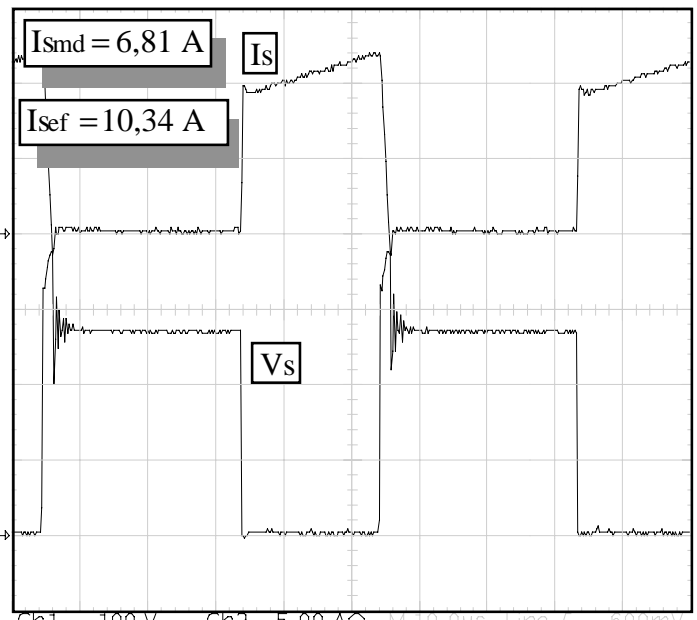

Figura 17: Tensão e corrente na chave S1. Escala: 300V/div; 8A/div; $10 \mu \mathrm{s} /$ div.

O fator de potência e a taxa de distorção harmônica (THD) da corrente de fase na rede elétrica são mostrados nas Figuras 20 e 21, respectivamente. Para a condição de plena carga o fator de potência obtido foi em torno de 0,96 , e a THD foi de $26 \%$. Para as mesmas condições de carga, o rendimento obtido foi em torno de $91 \%$ (Figura 22). As principais causas das perdas no conversor estão praticamente 
concentradas nos seguintes elementos: componentes magnéticos e filtro capacitivo, retificador de saída, e o emprego da técnica de comutação dissipativa.

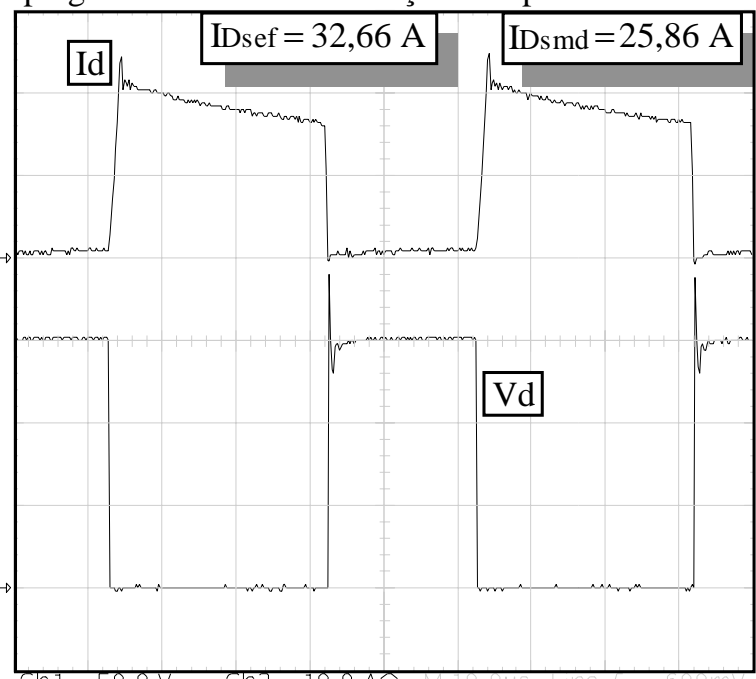

Figura 18: Tensão e corrente no diodo Ds.

Escala: $150 \mathrm{~V} / \mathrm{div} ; 25 \mathrm{~A} / \mathrm{div} ; 10 \mu \mathrm{s} / \mathrm{div}$.

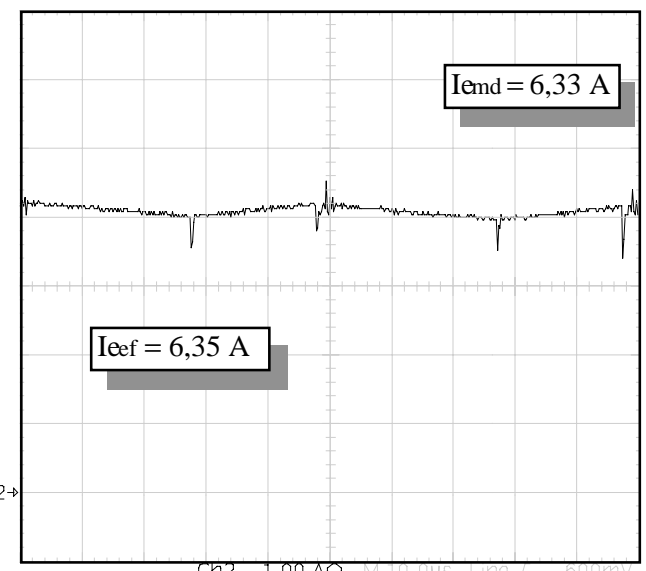

Figura 19: Corrente no indutor de entrada.

Escala: $1,5 \mathrm{~A} / \mathrm{div} ; 10 \mu \mathrm{s} / \mathrm{div}$.

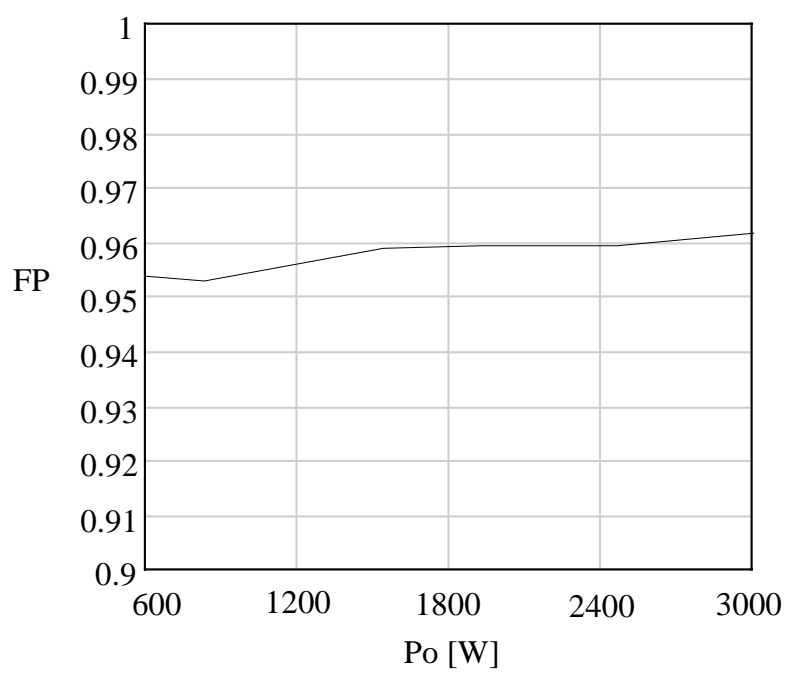

Figura 20: Comportamento do fator de potência.
Verifica-se que as figuras oriundas dos osciloscópios apresentam diferenças entre os valores de escala de tensão ou corrente e as legendas; isso porque foram usados transduto res, tendo em vista que os equipamentos não permitiam, de forma direta, obter-se as medidas feitas em bancada.

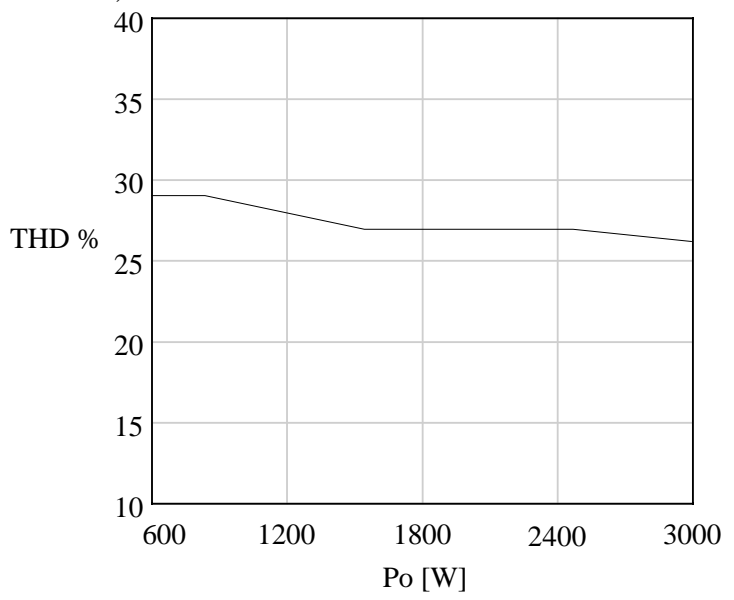

Figura 21: Taxa de distorção harmônica (THD) da corrente de fase na entrada do retificador.

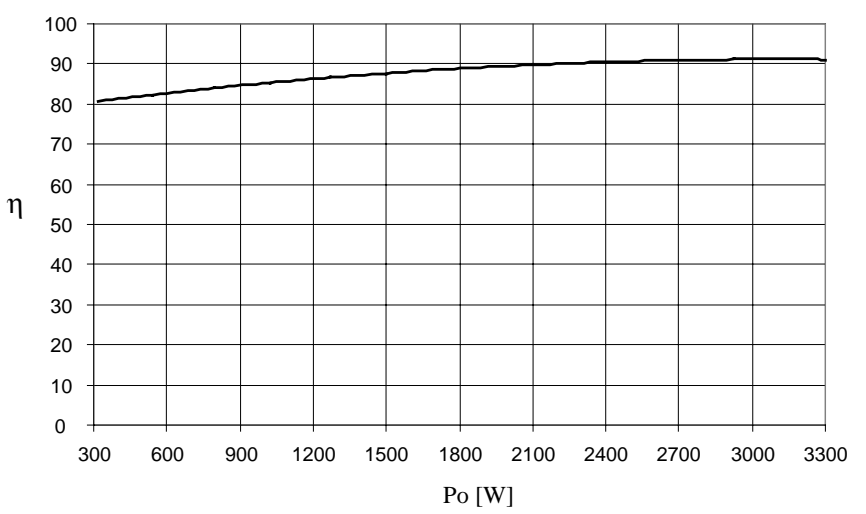

Figura 22: Curva de rendimento do protótipo de 3,0 kW.

A alta TDH da corrente de entrada presente neste circuito, é uma das características inerentes desta topologia, o que representa uma certa desvantagem da mesma. Contudo, é importante salientar que no nosso caso específico a tensão fornecida pela rede é altamente poluída, apresentando uma TDH na ordem de $2,6 \%$ a 3,0\%. É óbvio que essa alta distorção contribui para uma maior degradação da TDH da corrente de entrada. A TDH da corrente pode ser melhorada através de uma técnica de modulação da corrente no lado CC e realimentando-a no lado CA [2]. Todavia, esse procedimento demanda algum custo, que deve ser levado em conta, dependendo dos objetivos da aplicação deste circuito.

\section{CONCLUSÕES}

O retificador trifásico baseado no conversor CC-CC Sepic mostrou-se bastante robusto e de fácil montagem. O fato de possuir apenas uma chave para controlar o fluxo de energia faz com que o circuito de comando seja bastante simples. No protótipo implementado utilizou-se apenas um integrador para controlar o ganho estático de tensão. O número reduzido 
de componentes da estrutura aumenta a sua confiabilidade, tornando-a extremamente atrativa para aplicações industriais. Além disso, não é necessário utilizar filtros entre a rede comercial e o retificador trifásico. Esta estrutura é particularmente empregada em aplicações onde a carga apresenta um comportamento de fonte de tensão. De acordo com os resultados obtidos, tem-se um conversor CA-CC com as seguintes características:

- Topologia simples e robusta;

- Proporciona correção do fator de potência operando no modo de condução contínua, o que a torna atrativa para aplicações em altas potências;

- Estrutura naturalmente isolada;

- Apresenta uma única chave controlada;

- Pode operar como elevador ou abaixador de tensão, proporcionando uma maior flexibilidade, para um maior número de aplicações;

- Permite controlar a tensão de saída com apenas um único estágio de processamento de energia.

Finalmente, com essas características, a estrutura proposta pode ser empregada em potências elevadas, para uma faixa bem variada de aplicações industriais, sem qualquer dificuldade.

\section{REFERÊNCIAS BIBLIOGRÁFICAS}

[1] A. R. Prasad, P.D. Ziogas and S. Manias. "An Active Power Factor Correction Technique for Three Phase Diode Rectifiers". Proc. IEEE - PESC'89, pp. 58-65.

[2] O. Huang and F. Lee, "Harmonic Reduction In a Single Switch Three-Phase Boost Rectifier with Order Harmonic Injected PWM,' in IEEE -PESC'96, pp.12661271.

[3] C. T. Pan \& T.C. Chen. "Step-up/down Three Phase AC to DC Converter with Sinusoidal Input Current and Unity Power Factor". IEEE Proc. Electron. Power Appl., Vol. 141, no 2, pp. 52-77, March 1994.

[4] L. Malesani et al. "Single-Switch Three-Phase AC/DC Converter with High Power Factor and Wide Regulation Capability”. Proc IEEE - PESC92', pp. 279-285, June/1992.

[5] B. Ignazia, "Unity Power Factor Battery Charger Regulated by LVI," in Power Quality Proc., Nov. 1990, pp.42-47.

[6] D. Simonetty, J. Sebastian, and J. Uceda, "Single-Switch Three-Phase Power Pre-Regulator Under Variable Switching Frequency and Discontinuous Input Current," in IEEE-PESC'93 Conf. Rec., June 1993, pp. 657-661.

[7] J. Pforr and L. Hobson, "A Novel Power Factor Corrected Single Ended Resonant Converter With Three Phase Supply," in IEEE-PESC'92 Conf. Rec., in June 1992, pp. 1369-1375.

[8] A.H. Oliveira. "Three-Phase Rectifier with High Power Factor Using a Continuous Conduction Mode Sepic DCDC Converter". Master Thesis, INEP/EEL/UFSC, Florianópolis-SC-Brasil, 1996.

\section{DADOS BIOGRÁFICOS}

Denizar Cruz Martins, nasceu em São Paulo, SP, em 24 de Abril de 1955. Formou-se em Engenharia Elétrica e obteve o título de Mestre em Engenharia Elétrica pela Universidade Federal de Santa Catarina, Florianópolis - SC em 1978 e 1981, respectivamente. Concluiu o Doutorado no INPT, Toulouse - França, em 1986. Atualmente é professor titular do Depto. de Engenharia Elétrica da Universidade Federal de Santa Catarina, Florianópolis - SC. O Prof. Denizar já publicou mais de 100 trabalhos científicos entre revistas e congressos nacionais e internacionais, realizou mais de 30 consultorias técnicas e obteve 02 patentes de invenção e um registro de software. Sua área de atuação compreende: desenvolvimento de conversores para tratamento de energia solar, com alta qualidade de energia, conversores de alta freqüência e simulação de Conversor Estáticos. É membro da SOBRAEP, da SBA e do IEEE.

Anderson Hideki de Oliveira, nasceu em Campo Mourão, Paraná, em 19 de julho de 1969. Formou-se em Engenharia Elétrica pela Universidade Federal de Santa Catarina (UFSC), em 1994. Recebeu o título de Mestre em Engenharia Elétrica pela mesma Universidade em 1996. Atualmente é professor no CEFET do Paraná. Suas áreas de interesse são: conversores de alta freqüência, correção de fator de potência e retificadores trifásicos para altas potências.

Ivo Barbi, nasceu em Gaspar (SC), em 1949. Formou-se em Engenharia Elétrica pela Universidade Federal de Santa Catarina - UFSC, em 1973. Em 1976 recebeu o título de Mestre pela mesma Universidade e em 1979 recebeu o título de Doutor pelo Institut National Polytechnique de Toulouse, França. Desde 1974 é professor da UFSC e atualmente professor titular do Departamento de Engenharia Elétrica. É membro fundador da SOBRAEP tendo sido seu primeiro presidente. Desde 1992, é Editor Associado na área de Conversores de Potência da IEEE Transactions on Industrial Electronics. Suas áreas de atuação compreendem modelagem, análise, projeto e aplicações de conversores estáticos operando em alta frequiência e correção de fator de potência de fontes de alimentação. 